\title{
Capacity After Stroke
}

\author{
G. F. ADAMS
}

British Medical fournal, 1971, 1, 91-93

The new knowledge and clearer concepts of the pathogenesis of cerebrovascular disease and of "order and disorder" in the cerebral circulation acquired in recent years have improved the possibilities of treatment, which may now be considered at four stages in the evolution of a stroke: by prevention, when factors contributing to "stroke-proneness" may be identified and offset; by anticipation of impending stroke and the use of new techniques to locate and treat disordered regional blood flow in the cerebral circulation; by medical or surgical modification of progressing stroke; and by rehabilitation of completed stroke. There are two phases of the latter: the first a period of intensive care required at onset to save life and ensure survival: the second, often prolonged, being the treatment required to obtain the best recovery possible for a disabled survivor.

\section{Assessment of Patients}

No two hemiplegic patients are quite alike, and the management of residual disability must conform to individual needs. It is not possible, therefore, to define a rigid pattern of care, but a systematic method of assessment is helpful as a guide to treatment and to estimate progress and prognosis.

Strokes are phenomena especially but of course not exclusively of middle life and old age, and they resemble other geriatric neurological disorders in seldom presenting with motor deficit alone. Almost always there is associated diffuse brain damage. This is because brain function depends on the endowment of brain cells inherited at birth and on the efficiency of the circulation maintaining their nutrition. As people grow older this efficiency may be impaired by defec-

Wakehurst House, Belfast City Hospital, Belfast, Northern Ireland G. F. ADAMS, M.D., F.R.C.P., Physician in Geriatrics

tive blood flow (in diseased or abnormal vessels), by impaired quality of the perfusing blood (in anaemia or disturbed water and electrolyte balance), or by altered blood constituents (especially changes contributing to thrombosis or embolism).

At the same time loss of neurones, or "fall-out" in the neuraxis-a phenomenon even of normal old age-may build up to pathological levels as a feature of senile or arteriosclerotic cerebral degeneration or of toxic disorders, and brain function may be correspondingly impaired. If, then, from one cause or another the defective cerebral circulation fails at some point the patient may have a stroke, but this dramatic presentation may obscure the evidence of underlying disorders such as intellectual deterioration, sensory deficit, or impaired postural control.

The first principle in the management of strokes is to look for signs of these associated disorders when assessing the extent of the damage; the second is to remember that the patient cannot be expected to have a better make-up after a stroke than he had before it, and that the necessary information about this will best be given by relatives who knew the patient before his illness or by those most closely in touch with him afterwards, whether relatives, nurses, or therapists.

\section{Criteria in Selection for Care}

The treatment of hemiplegia is based on two estimates-one diagnostic, the other an estimate of capacity. The precise cause of a stroke must be diagnosed at the onset so that an associated emergency, such as subdural haematoma or coronary infarction, will not be overlooked; but continuous review of clinical findings is necessary throughout recovery also, because time is so often the critical factor in neurological diagnosis. Strokes attributable to cerebral tumours illustrate this. The distinction of these from strokes caused by vascular accidents is of more than academic importance. Survival for months or years is to be expected after recovery from a vascular accident, and resolute efforts must be 
made to overcome the handicaps of a recovery which is delayed. On the other hand, the earlier it is possible to identify a tumour when it is the cause of hemiplegia the better. Apart from the possibilities of successful surgery, the patient with an inoperable tumour may be spared long weeks of exhausting physical effort made in vain, and attention may be concentrated instead on essential terminal care, sedation, and comforts.

In the second estimate the emphasis is on capacity-on remaining ability and reserves, and not on disability. We do not commiserate too much with the elderly patient on what he has lost; rather do we congratulate him on what he has left, encouraging and helping him to make the most of it. This positive attitude is reflected in the selection of hemiplegic patients for rehabilitation. The following criteria are defined not to reject patients from treatment but to accept them for it.

\section{STRENGTH AND ENDURANCE}

This is exercise tolerance, but is expressed as strength and endurance to underline what is important to the elderly hemiplegic. Muscular weakness is commonplace in old people, and their tolerance of effort varies from day to day, so that it is necessary to known how they responded to exercise and what their staying-power was before the stroke.

Some patients have the added handicaps of arthritis, amputation, or respiratory insufficiency. Others are at risk of low blood-pressure states owing to defective baroreceptor mechanisms, coronary infarction (associated perhaps with $20 \%$ of strokes), or adverse reactions to sedatives, especially to chlorpromazine and allied phenothiazine derivatives. Their distress may be overlooked unless an observant physiotherapist or nurse notices the limits on exercise imposed by shortness of breath or impending syncope.

\section{MOTIVATION}

To estimate motivation, one has to know about the patient's emotional and intellectual make-up, initiative, and resilience before the stroke. Sometimes it seems that patients of good educational and professional attainment, who might be expected to make the best recovery from strokes, respond worst, as if unable to accept their limitations. These include the patients who simply "don't want to do it," whatever it may be.

\section{MENTAL CAPACITY}

One does not attempt to estimate mental capacity by ambitious intelligence tests. What is required is a convenient yardstick to measure progress from week to week in patients whose intellectual capacities vary from one to another and from day to day, even without such added handicaps as clouded consciousness, dysphasia, depression, or denial of disease. The following are practical criteria.

Level of consciousness, estimated from an assessment of comprehension, concentration, insight (concern), orientation, and perseveration. A patient may give a superficial impression of quick recovery from coma or stupor at the onset of a stroke when, in fact, tests will show him still to be considerably retarded, inattentive, and disorientated. Despite a patient's cheerful air of unconcern, these handicaps may persist for many weeks, and allowances must be made for them in the attitudes of those responsible for treatment. Routine historytaking and examination will provide some information on these points, but the patient who is clouded, withdrawn, or dysphasic may have to be primed with specific questions before the level and accuracy of responsiveness can be determined.
Communication-Problems of reception (input), of expression (output), and of central integration (comprehension?). This oversimplifies the speech problems associated with hemiplegia, but it is helpful to think of them in this way so as to distinguish patients who cannot express themselves intelligibly but can comprehend and act on spoken or written instructions, from those who speak but who do not comprehend properly and from those who do neither but may still respond to mime.

Memory.-This is often defective in old age, but loss of very recent memory may be a specific problem in the treatment of some hemiplegics. They may be unable to retain instructions or advice for more than a few minutes, and painstaking repetition is required, sometimes for weeks, before there is any response.

Behaviour-spontaneity, mood (depression), continence. Here again it is important to know what kind of person the patient was before attributing a lack of responsiveness or depression to brain damage. As in so many other aspects of recovery one is dependent on those attending the patient constantly by day and by night for information about behaviour. When inquiring about recovery from incontinence it is important to make sure that "dry" nights or days really are the result of improvement in the patient's own control and not simply a tribute to good nursing. Persistent incontinence in the absence of local causes is a discouraging prognostic sign. It may be part of a picture of deteriorating intellect, or it may be associated with cerebral damage in the frontal area of the right (non-dominant) hemisphere. Recovery from incontinence related to lesions in this area seems of ten to be unduly prolonged or, if associated with severe sensory deficit and anosognosia, to remain intractable.

Performance-reasoning, dexterity, critical faculties, selfcare. Impressions on these points may be volunteered by nurses and others, but more precise estimates of capacity are given by simple tests, including writing or drawing (and a felt pen gives better results than a pencil or a ball-point pen), and by the use of toys such as Kohs's blocks, a peg-board, or a posting-box.

\section{MOTOR DEFICIT}

Motor deficit includes weakness, spasticity, and contractures. It is not necessary to consider this condition in detail, because it has always attracted the most attention in rehabilitation, though sometimes this emphasis may be misguided. The essentials are to prevent contracture; to concentrate in the early stages on mobility of proximal muscle groups (shoulder and hip rather than hand and foot); to restore balance and confidence when moving; to avoid over-use of the "good" side at the expense of the hemiplegic limbs; and to strive, if necessary for months, to obtain a polished rhythmical gait and not accept too early or too readily the stilted awkwardness of the traditional hemiplegic hobble.

\section{SENSORY DEFICIT}

This includes vision, hearing, proprioception, discrimination, and cortical integration-disorders of body image. Defective eyesight and hearing are obvious sources of difficulty in rehabilitation, and they are apt to be overlooked unless it is routine practice to inquire whether the patient needs glasses or a hearing aid. Homonymous hemianopsia may be expected to lessen if not to recover completely, and meanwhile the patient should be approached from the side with normal vision and have his locker and other necessaries where he can see them. Some patients are not even aware that they have lost one-half of their field of vision, so that their lack of concern about poor performance can easily be appreciated. These patients must face difficulties analogous to those expe- 
rienced by some old people after cataract operations, when, as Trevor-Roper ${ }^{1}$ said, the pebble-lenses needed after the operation surround them by a distorted world which squirms about with every eye movement, while "faces pop in and out of the blind area with the annoying insolence of a jack-in-thebox." He believes that many a frail psyche faced with the choice would prefer "to grope its way through life rather than cope with this formidable new visual world." The hemiplegic patient does not have any choice, and the "frail psyche" many are left with needs all possible support and consideration to make life tolerable.

Patients isolated by deafness sometimes become introspective, withdrawn, depressed, or even delusional. Their inaccessibility may make heavy demands on the patience of those who are trying to help them. It should be remembered that distortion of the quality of sound and intolerance of amplification are common in perceptive deafness, and patients should not be shouted at; instead they must be addressed in clear, explicit speech, from a position which permits them to lipread.

The intimate association between sensation and motor function is well known. Defective proprioception denies the patient an assured knowledge of joint position and movement, disrupts the feed-back mechanisms maintaining muscle length and tension, and upsets the control of balance, control of the arm, and control of walking. Disordered discriminative sensation at cortical level interferes with touch localization and awareness of shape, size, form, and texture. This has distinctive effects on the precision use of the hand and fingers, contributing perhaps to forms of apraxia. Impaired cortical integration upsets consciousness of body image, creating problems of neglect of the hemiplegic limbs (despite effective recovery of voluntary power) or even more bizarre symptoms, such as loss of awareness of paralysis or even denial of ownership of limbs.

Clinical patterns of disordered sensation are variable not only from one patient to another but also in the same patient from time to time, when levels of awareness and atten- tiveness are patchy. This may account for variable levels of performance, and adds to the difficulties of prognosis in the early stages of recovery.

\section{POSTURAL CONTROI}

The patient who cannot sit up with confidence cannot stand, and the patient who cannot stand reliably cannot walk.

There are two pathways of postural control which may be affected in cerebrovascular accidents, either vestibular/cerebellar co-ordination in the brain stem or the visuo-spatial integrating systems of the parietal cortex. These sensitive mechanisms are probably dependent on a complex network of intracerebral and basal ganglial connexions, and it is not surprising that some disorder of postural fixation and balance, temporary or more persistent, is commonplace in recovery from strokes.

\section{Conclusion}

Use of the foregoing criteria sets a pattern of assessment of capacity after a stroke which can indicate when to treat, what to treat, and how to treat it, matching the management of the patient with progress past the milestones of recovery from sitting to standing, then to walking, and finally to social reliability. In the early years, before this systematic follow-up was applied to treatment, about equal numbers of the patients who survived severe or moderately severe strokes recovered or became long-stay failures. Results now show a $30 \%$ difference in favour of recovery, ${ }^{2}$ a tribute to the more imaginative and constructive efforts being made to overcome these mental barriers to recovery from strokes.

\section{References}

1 Trevor-Roper, P. D., British Medical fournal, 1970, 3, 33.

2 Adams, G. F., Gerontologia Clinica. In press.

\section{Coronary Artery Disease}

\section{I-Management of the Acute Episode}

\section{B. L. PENTECOST}

British Medical fournal, 1971, 1, 93-95

The current treatment of acute myocardial infarction is designed to deal with two well-recognized features of its natural history: firstly, a peak mortality within the first few hours of the episode ${ }^{2}$; and, secondly, a high incidence of deaths related to arrhythmias rather than to massive injury of the myocardium. There has, therefore, been a general trend

The General Hospital, Birmingham 4

B. L. PENTECOST, M.D., M.R.C.P., Consultant Physician

towards early hospital admission, particularly in the younger good-risk patient. Conversely, in an elderly patient with recurrence of infarction together with pre-existing chronic failure the main problems are likely to be those of severe mechanical failure. In this case home treatment may under certain conditions be preferable to hospital care. The early occurrence of arrhythmias reduces the therapeutic potential of the hospital coronary care unit and has led to the development of mobile units in some centres. ${ }^{34}$ The achievements of mobile and hospital units alike have been mainly in treating arrhythmias rather than those complications resulting from severe myocardial injury. 